\title{
Minimum infusion rate and adrenocortical function after continuous infusion of the novel etomidate analog ET-26-HCI in rats
}

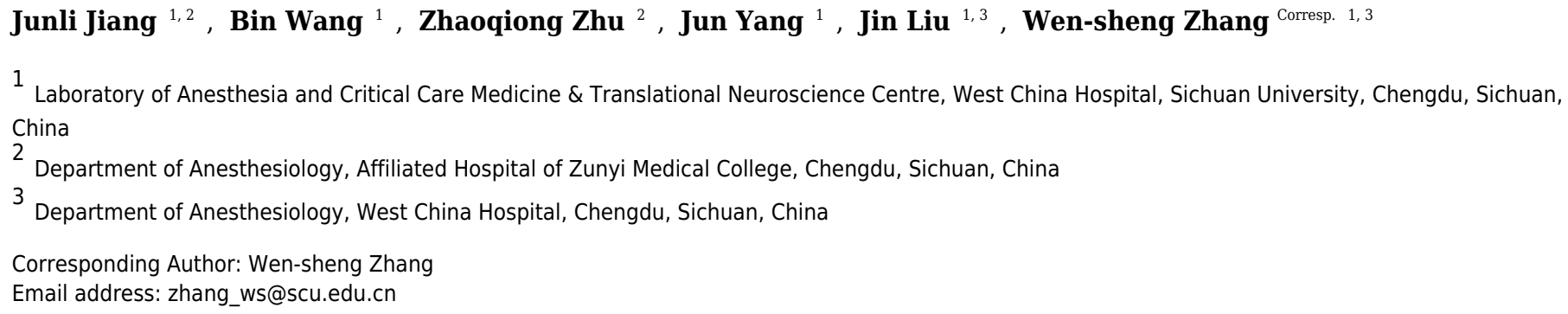

Background. Because etomidate induces prolonged adrenal suppression, even following a single bolus, its use as an infused anesthetic is limited. Our previous study indicated that a single administration of the novel etomidate analog methoxyethyletomidate hydrochloride $(\mathrm{ET}-26-\mathrm{HCl})$ shows little suppression of adrenocortical function. The aims of the present study were to (1) determine the minimum infusion rate of ET-26- $\mathrm{HCl}$ and compare it with those for etomidate and cyclopropyl-methoxycarbonylmetomidate (CPMM), a rapidly metabolized etomidate analog that is currently in clinical trials and (2) to evaluate adrenocortical function after a continuous infusion of ET-26- $\mathrm{HCl}$ as part of a broader study investigating whether this etomidate analog is suitable for long infusion in the maintenance of anesthesia. Method. The up-and-down method was used to determine the minimum infusion rates for ET-26-HCl, etomidate and CPMM. Sprague-Dawley rats $(n=32)$ were then randomly divided into four groups: etomidate, ET-26- $\mathrm{HCl}, \mathrm{CPMM}$, and vehicle control. Rats in each group were infused for 60 min with one of the drugs at its predetermined minimum infusion rate. Blood samples were drawn initially and then every 30 min after drug infusion to determine the adrenocorticotropic hormone-stimulated concentration of serum corticosterone as a measure of adrenocortical function. Results. The minimum infusion rates for etomidate, ET-26- $\mathrm{HCl}$ and CPMM were $0.29,0.62$, and 0.95 $\mathrm{mg} / \mathrm{kg} / \mathrm{min}$, respectively. Compared with controls, etomidate decreased serum corticosterone, as expected, whereas serum corticosterone concentrations following infusion with the etomidate analogs $\mathrm{ET}-26-\mathrm{HCl}$ or CPMM were not significantly different from those in the control group. Conclusion. The corticosterone concentrations tended to be reduced for the first hour following $\mathrm{ET}-26-\mathrm{HCl}$ infusion (as compared to vehicle infusion); however, this reduction did not reach statistical significance. Thus, further studies are warranted examining the practicability of using $\mathrm{ET}-26-\mathrm{HCl}$ as an infused anesthetic. 
1 Minimum infusion rate and adrenocortical function after continuous infusion of the novel etomidate analog $\mathrm{ET}-26-\mathrm{HCl}$ in rats

3 Junli Jiang ${ }^{1,3 \#}$, Bin Wang ${ }^{1 \#}$, Zhaoqiong Zhu ${ }^{3}$, Jun Yang ${ }^{1}$, Jin Liu ${ }^{1,2}$, Wensheng Zhang ${ }^{1,2 *}$,

$4{ }^{1}$ Laboratory of Anesthesia and Critical Care Medicine, Translational Neuroscience Center, West

5 China Hospital, Sichuan University, Chengdu 610041, Sichuan, P. R. China;

6 '2Department of Anesthesiology, West China Hospital, Sichuan University, Chengdu 610041, 7 Sichuan, P. R. China;

$8{ }^{3}$ Department of Anesthesiology, Affiliated Hospital of Zunyi Medical University, Zunyi 563000,

9 Guizhou, P. R. China.

\#These authors contributed equally to this work.

12 Address Correspondence to Dr. Wensheng Zhang:

13 Laboratory of Anesthesia and Critical Care Medicine, Translational Neuroscience Center, West

14 China Hospital, Sichuan University, Chengdu 610041, Sichuan, P. R. China.

15 Tel: $+86-2885164144$

16 E-mail: zhang_ws@scu.edu.cn 


\section{Abstract}

19

20

21

22

Background. Because etomidate induces prolonged adrenal suppression, even following a single bolus, its use as an infused anesthetic is limited. Our previous study indicated that a single administration of the novel etomidate analog methoxyethyletomidate hydrochloride (ET-26-HCl) shows little suppression of adrenocortical function. The aims of the present study were to (1) determine the minimum infusion rate of ET-26- $\mathrm{HCl}$ and compare it with those for etomidate and cyclopropyl-methoxycarbonylmetomidate (CPMM), a rapidly metabolized etomidate analog that is currently in clinical trials and (2) to evaluate adrenocortical function after a continuous infusion of ET-26- $\mathrm{HCl}$ as part of a broader study investigating whether this etomidate analog is suitable for long infusion in the maintenance of anesthesia.

Method. The up-and-down method was used to determine the minimum infusion rates for ET-26$\mathrm{HCl}$, etomidate and CPMM. Sprague-Dawley rats $(\mathrm{n}=32)$ were then randomly divided into four groups: etomidate, ET-26-HCl, CPMM, and vehicle control. Rats in each group were infused for 60 min with one of the drugs at its predetermined minimum infusion rate. Blood samples were drawn initially and then every $30 \mathrm{~min}$ after drug infusion to determine the adrenocorticotropic hormone-stimulated concentration of serum corticosterone as a measure of adrenocortical function.

Results. The minimum infusion rates for etomidate, ET-26- $\mathrm{HCl}$ and CPMM were 0.29, 0.62, and $0.95 \mathrm{mg} / \mathrm{kg} / \mathrm{min}$, respectively. Compared with controls, etomidate decreased serum corticosterone, as expected, whereas serum corticosterone concentrations following infusion with the etomidate 
38 analogs ET-26-HCl or CPMM were not significantly different from those in the control group.

39 Conclusion. The corticosterone concentrations tended to be reduced for the first hour following

40 ET-26-HCl infusion (as compared to vehicle infusion); however, this reduction did not reach

41 statistical significance. Thus, further studies are warranted examining the practicability of using

42 ET-26- $\mathrm{HCl}$ as an infused anesthetic.

\section{Introduction}

Etomidate, one of the most frequently used intravenous anesthetics, has many favorable properties, such as its ability to maintain hemodynamic stability and generate low incidences of respiratory depression and anaphylaxis. However, the adrenocortical insufficiency caused by etomidate restricts its clinical applications. Several studies have shown that as little as a single administration of etomidate may induce adrenocortical insufficiency, and the increased propensity for this may last $48 \mathrm{~h}$ after the administration of etomidate (Hildreth et al. 2008; Tekwani et al. 2008).

In the 1980s, etomidate was infused in a continuous manner in critical patients to maintain sedation; however, studies later showed that this dosage regimen increased mortality (Ledingham \& Watt 1983; Watt \& Ledingham 1984). Pharmacologists have spent years attempting to develop new etomidate analogs in their search for a drug that retains the desirable properties of etomidate but does not cause adrenocortical insufficiency. We recently reported on ET-26-HCl, a promising compound selected from dozens of other etomidate analogs that were designed using our synthesis strategy, showing that ET-26-HCl effectively produces reversible anesthesia, and that a signal 
58

59

60

61

62

administration does not significantly decrease plasma corticosterone levelsin beagle dogs (Yang et al. 2017). The primary aim of the present study was to evaluate the effect of ET-26- $\mathrm{HCl}$ on adrenal function after a continuous infusion.

\section{Materials and Methods}

Animals and materials: All animal protocols used in the present study were approved by the Ethics Committee of the West China Hospital, Sichuan University, China (ethics approval No. 2015015A; date: 28/12/2012). Sprague Dawley rats weighing 225-350 grams were purchased from Chengdu Dassy Biological Technology Co. Ltd. (Chengdu, China) and cared for in accordance with the the Canadian Council on Animal Care's Guide to the Care and Use of Experimental Animals (Vol. 1 2nd ed., 1993). Five animals per cage were housed under standard conditions at a temperature of $22^{\circ} \mathrm{C}$ and a humidity of $60 \%$ and with standard laboratory rat chow and water. The animals were allowed to acclimatize for 1 week.

Etomidate $(2 \mathrm{mg} / \mathrm{mL})$ formulated as an emulsion was purchased from B. Braun Melsungen AG, and propofol $(10 \mathrm{mg} / \mathrm{mL})$ formulated as an emulsion was purchased from AstraZeneca. The Laboratory of Anesthesia and Critical Care Medicine (West China Hospital, Sichuan University, China) synthesized ET-26-HCl using a previously published approach; ET-26-HCl was formulated as an aqueous solution $(10 \mathrm{mg} / \mathrm{mL})$ with $35 \%$ propylene glycol and then diluted with normal saline (0.9\%; Kelun Pharmaceutical Co., Ltd.) to $6 \mathrm{mg} / \mathrm{mL}$. Cyclopropyl-methoxycarbonylmetomidate (CPMM) was also synthesized by our laboratory according to the issued patent (patent No., 
US9156825B2) and formulated as an aqueous solution $(8 \mathrm{mg} / \mathrm{mL})$ with $20 \%$ sulfobutylether- $\beta$ cyclodextrin (Campagna et al. 2014).

An infusion pump (Sino Medical-device Technology Co., Ltd.) was used to administer the sedatives and hypnotics, and a homoeothermic blanket was used to maintain the body temperature of the rats at $36-38^{\circ} \mathrm{C}$ while they were anesthetized. A pulse oximeter placed on the upper right hind leg was used for monitoring oxygen saturation. Heart rate and rhythm and the respiratory rate were monitored with a BL-420S biological data acquisition and analysis system (Taimeng Software Co. Ltd., Chengdu, China). Oxygen (100\%) was delivered at a rate of $2 \mathrm{~L} / \mathrm{min}$ to each anesthetized rat through a face mask connected to a coaxial circuit.

\section{Determination of the minimum infusion rate}

Rats $(n=60)$ were randomly assigned to three groups that received either etomidate, ET-26-HCl, or CPMM.

A 22-gauge catheter was inserted into the caudal vein of the rat for drug infusion. The minimum infusion rate (MIR) for each anesthetic was determined as previously described (Ge et al. 2012). The initial infusion rates of etomidate and CPMM were based on those that had been determined in previous studies (Ge et al. 2013). Based on our preliminary experimental results indicating that ET-26- $\mathrm{HCl}$ was one-third as potent a hypnotic as etomidate, the initial infusion rate of ET-26-HCl was three times greater than that of etomidate. The first subject in each group was administered the selected initial continuous infusion rate for one drug for $40 \mathrm{~min}$. Then the animal's response to noxious stimulation was determined. A painful stimulus was provided by clamping the tail with 
98 an alligator clip and rolling the clamp at $1-2 \mathrm{~Hz}$ for $60 \mathrm{~s}$ or until the rat exhibited a purposeful

99 response. Based on the presence or absence of purposeful movements of the extremities, the

100 infusion rate for that drug was increased or decreased by $10 \%$ ( $\mathrm{Li}$ et al. 2012) in the next rat and

101 held constant for 40 minutes before the tail-clamping stimulation was repeated. The response of

102 the rats to the painful stimulus was judged to be either negative or positive. When the rat showed

103 a gross purposeful movement (e.g., limbs retracted or head twisted), the response would be

104 considered positive, and the infusion rate of the drug for the next rat was increased. Conversely,

105 when rats made no gross purposeful movement, the infusion rate for the next rat was reduced. A

106 change in the direction of the response from negative to positive or positive to negative was defined

107 as a pair, and the stimulation was repeated at different infusion rates until five pairs of responses

108 were recorded. The MIR was calculated as the average of these five mean values.

109 Evaluation of adrenocortical function

110 The infusion protocol for each rat began at 8:30-9:00 a.m.to minimize natural changes in

111 corticosterone levels. After the rats were weighed and the caudal vein was catheterized,

112 dexamethasone $(0.2 \mathrm{mg} / \mathrm{kg})$ was administered. Two hours later, the baseline blood sample was

113 drawn. Rats $(\mathrm{n}=32)$ were then randomly divided into the following four groups, with 8 rats in

114 each group: etomidate, ET-26-HCl, CPMM, or propylene glycol vehicle control, and the infusion

115 protocol started (see Fig. 1A). The infusion rate in each group was the corresponding

116 predetermined MIR for that compound, and the drug infusion persisted for 60 min. After 30

117 minutes of drug infusion, adrenocorticotropic hormone (ACTH) was administered intravenously, 
118 and this was repeated every $30 \mathrm{~min}$. The second blood sample was collected at the end of the 119 infusion, and then blood samples were drawn every $30 \mathrm{~min}$ for $3.5 \mathrm{~h}$. Blood samples 120 (approximately $0.2 \mathrm{~mL}$ each) were kept at room temperature for 10 to $60 \mathrm{~min}$, until coagulation, 121 and then centrifuged at 3,500 gravity $(g)$ for $5 \mathrm{~min}$. The supernatant was transferred to a clean 122 Eppendorf centrifuge tube and subjected to a second centrifugation (3500 rpm for $5 \mathrm{~min}$ ). After 123 the second centrifugation, the supernatant was removed again, and a final high-speed 124 centrifugation was performed to remove all red blood cells and particulate. The serum was transferred to a clean tube and immediately placed at $-20^{\circ} \mathrm{C}$. The corticosterone concentration in each serum sample obtained at the various time points was quantified within 1-2 days using an enzyme-linked immunosorbent assay (ELISA kit, R\&D Systems) and a 96-well plate reader. $36-38^{\circ} \mathrm{C}$, and oxygen was continuously provided at $2 \mathrm{~L} / \mathrm{min}$.

\section{Statistical analysis}

All data are presented as the mean \pm standard deviation. For comparisons of serum corticosterone concentrations after infusion of vehicle and test compounds, a one-way analysis of variance was conducted followed by Tukey's honest significant difference test or the Tamhane test. A value of $P<0.05$ was considered statistically significant. All statistical analyses were carried out using the Statistical Package for Social Sciences version 21.0 software (Chicago, IL, USA). Figure preparation and curve fitting was performed with Prism version 5.0 (GraphPad Software, Inc., La Jolla, CA, USA). 


\section{Results}

\section{Minimum infusion rate of each drug}

141 The MIRs of etomidate, CPMM, and ET-26-HCl were 0.29, 0.95, and $0.62 \mathrm{mg} / \mathrm{kg} / \mathrm{min}$, 142 respectively (Table 1$)$.

\section{Evaluation of adrenocortical function}

144 The baseline concentrations of serum corticosterone (before the start of the drug infusion) were not significantly different among the four groups and averaged $185.86 \pm 68.66 \mathrm{ng} / \mathrm{mL}$. However, serum corticosterone concentrations increased over time in all groups after drug infusion and

ACTH stimulation. Compared with the control group, rats administered etomidate demonstrated significantly lower corticosterone concentrations at $60 \mathrm{~min}$, whichis the end of the drug infusion, as well as 90, 120, 150, and $240 \mathrm{~min}$ after the begin of drug infusion. Compared with the etomidate group, rats administered ET-26-HCl showed significant differences 90, 120, 180, 210, and 240 min after begin of drug infusion. Compared with rats administered CPMM, rats administered etomidate showed significant differences in serum corticosterone concentrations at the end of the significantly different from each other at any time (Fig. 1B). 


\section{Discussion}

158 In the present study, we evaluated the MIRs of etomidate, ET-26-HCl, and CPMM by recording 159 in anesthetized rats either a positive $(+)$ or no $(-)$ reaction to a painful stimulus, an up-and-down 160 design method previously described ( $\mathrm{Li}$ et al. 2012). Each hypnotic was administered intravenously for 40 min to determine its MIR because our preliminary study showed that the halflife of ET-26- $\mathrm{HCl}$ was 6-7 min, the longest of the three hypnotics used in the present study, and because it is generally acknowledged that the in vivo plasma concentration of drugs continuously infused at a constant rate reaches equilibrium at 4-5 half-lives. We determined that the MIR for etomidate was $0.285 \mathrm{mg} / \mathrm{kg} / \mathrm{min}$, for $\mathrm{ET}-26-\mathrm{HCl}$ it was $0.62 \mathrm{mg} / \mathrm{kg} / \mathrm{min}$, and for CPMM it was $0.95 \mathrm{mg} / \mathrm{kg} / \mathrm{min}$. These results suggested that the anesthetic efficacy of ET-26-HCl was approximately one-half to one-third of that for etomidate, which is consistent with the results of our previous study (Yang et al. 2017). In addition, the MIR of CPMM found in the present study was consistent with the results of Ge and colleagues, which suggested that the immobilizing $\mathrm{ED}_{50}$ (effective dose for 50 percent of the group) of CPMM is $0.89 \pm 0.18 \mathrm{mg} / \mathrm{kg} / \mathrm{min}$ (Ge et al. 2012). These authors also found that the total doses of etomidate and CPMM needed in a 2-h closed-loop infusion protocol to maintain an $80 \%$ electroencephalographic burst suppression ratio are 36 $\mathrm{mg} / \mathrm{kg}$ and $143 \mathrm{mg} / \mathrm{kg}$, respectively, indicating that the average infusion rates for these hypnotics are $0.3 \mathrm{mg} / \mathrm{kg} / \mathrm{min}$ and $1.19 \mathrm{mg} / \mathrm{kg} / \mathrm{min}$, respectively. This infusion rate for etomidate is consistent with the rate found in the present study, while the rate for CPMM in the present study is slightly lower than that observed in the previous study. Thus, we speculate that all rats in the three groups used in the present study were at a similar depth of anesthesia. 
178

179

180

181

182

183

184

185

186

187

188

189

190

191

192

193

194

195

196

197

198

After determining the MIRs, we next examined the effects of etomidate, ET-26-HCl, and CPMM

continuously infused for $1 \mathrm{~h}$ and inducing the same anesthesia depth on serum corticosterone concentrations. Compared with those in control rats, ACTH-stimulated serum corticosterone concentrations were significantly decreased by etomidate, while those following ET-26-HCl or CPMM administration were not associated with a significant difference. When ACTH-stimulated serum corticosterone concentrations in rats administered etomidate were compared with those in rats infused with vehicle, ET-26-HCl or CPMM, all time points examined after the drug infusions, except $180 \mathrm{~min}$ and $240 \mathrm{~min}$, show significant differences with etomidate. The corticosterone concentrations tended to be reduced for the first hour following ET-26-HCl infusion (as compared to vehicle infusion); however, this reduction did not reach statistical significance. We concluded that ET-26-HCl does not induce obvious inhibition of adrenal function.

The safe dosage range of etomidate has been diminishing owing to its inhibition of adrenocortical function, as the suppression following even a single bolus may last $72 \mathrm{~h}$ (Molenaar et al. 2012).

This inhibition is mainly the result of the high-affinity binding between the basic nitrogen in the imidazole ring of etomidate and the heme iron on 11ß-hydroxylase (den Brinker et al. 2008; Fellows et al. 1983; Shanmugasundararaj et al. 2013). In the 1980s, etomidate was used as a sedative for critically ill patients; however, in 1983, Watt and colleagues found that the continuous infusion of etomidate may increase mortality, and they speculated that the increased mortality is mainly caused by adrenocortical suppression. A series of studies later verified this speculation and recommended not to blindly administer etomidate to critical patients (Morris \& McAllister 2005). However, no other anesthetic currently possesses the characteristics of etomidate, such as to the 
199

200

201

202

203

204

205

206

207

208

209

210

211

212

213

214

215

216

217

218

219

ability to maintain stable hemodynamics, especially in aged or critically ill patients. Therefore, researchers have devoted much effort to develop new etomidate analogs that preserve the advantages but reduce the disadvantages of etomidate. There are at least two ways to achieve this goal. The first method involves designing a series of analogs that are rapidly metabolized so that adrenocortical inhibition stops soon after the administration is discontinued. With this in mind, MOC-etomidate and CPMM were designed by researchers at the Massachusetts General Hospital. Among these compounds, CPMM showed the greatest promise for development (Campagna et al. 2014; Cotten et al. 2010; Cotten et al. 2009; Pejo et al. 2012; Santer et al. 2015). The second method involves changing the molecular structure of etomidate to minimize adrenocortical suppression. It is widely acknowledged that the primary mechanism of adrenocortical suppression is through the interaction between the basic nitrogen in the imidazole ring of etomidate and the heme iron of 11ß-hydroxylase (Gay et al. 2009; Ouellet et al. 2008; Roumen et al. 2007). Several studies have shown that anesthesia efficacy is significantly decreased when the basic nitrogen is replaced with other chemical groups, such as in carboetomidate, which has an anesthesia potency approximately one-seventh of that for etomidate (Cotten et al. 2010). However, Atucha and colleagues suggested that the imidazole carboxylic acid ester side chain of etomidate affects both anesthetic potency and adrenocortical function (Atucha et al. 2009). The design of ET-26-HCl is based on modifications of this side chain, and our previous study showed that ET-26-HCl produces definite and reversible anesthesia in beagle dogs. In the present study, no significant difference was observed in the serum corticosterone concentration after the continuous infusion of ET-26$\mathrm{HCl}$ or vehicle, suggesting that any adrenocortical suppression induced by ET-26-HCl would be 
220 lower than that caused by etomidate. These results also indicated that new analogs may be

221 developed by means other than using soft analogs.

222

223 Conclusion

224 The method to evaluate minimum infusion rate of present study is feasible, and it could maintain 225 a similar anesthesia depth of all drugs. The corticosterone concentrations tended to be reduced for 226 the first hour following ET-26-HCl infusion (as compared to vehicle infusion) was mainly because 227 of the molecular structure of ET-26-HCl still have some depression to the release of corticosterone;

228 however, this reduction did not reach statistical significance. Thus, further studies are warranted 229 examining the practicability of using ET-26- $\mathrm{HCl}$ as an infused anesthetic.

232 Abbreviations

233 MIR: minimum infusion rate

234 ACTH: adrenocorticotropic hormone

235 ET-26-HCl: methoxyethyletomidate hydrochloride

236 CPMM: cyclopropyl-methoxycarbonylmetomidate

\section{References}


241

242

243

244

245

246

247

248

249

250

251

252

253

254

255

256

257

258

259

260

261

262

263

264

265

266

267

268

269

270

271

272

273

274

275

276

277

278

279

280

281 derivatives at the $\mathrm{GABA}(\mathrm{A})$ receptor: Comparison with binding to 11beta-hydroxylase. Bioorg Med Chem Lett 19:4284-4287. 10.1016/j.bmcl.2009.05.065

Campagna JA, Pojasek K, Grayzel D, Randle J, and Raines DE. 2014. Advancing novel anesthetics: pharmacodynamic and pharmacokinetic studies of cyclopropyl-methoxycarbonyl metomidate in dogs. Anesthesiology 121:1203-1216. 10.1097/aln.0000000000000416

Cotten JF, Forman SA, Laha JK, Cuny GD, Husain SS, Miller KW, Nguyen HH, Kelly EW, Stewart D, Liu A, and Raines DE. 2010. Carboetomidate: a pyrrole analog of etomidate designed not to suppress adrenocortical function. Anesthesiology 112:637-644. 10.1097/ALN.0b013e3181cf40ed

Cotten JF, Husain SS, Forman SA, Miller KW, Kelly EW, Nguyen HH, and Raines DE. 2009. Methoxycarbonyletomidate: a novel rapidly metabolized and ultra-short-acting etomidate analogue that does not produce prolonged adrenocortical suppression. Anesthesiology 111:240-249. 10.1097/ALN.0b013e3181ae63d1

den Brinker M, Hokken-Koelega AC, Hazelzet JA, de Jong FH, Hop WC, and Joosten KF. 2008. One single dose of etomidate negatively influences adrenocortical performance for at least $24 \mathrm{~h}$ in children with meningococcal sepsis. Intensive Care Med 34:163-168. 10.1007/s00134-007-0836-3

Fellows IW, Bastow MD, Byrne AJ, and Allison SP. 1983. Adrenocortical suppression in multiply injured patients: a complication of etomidate treatment. Br Med J (Clin Res Ed) 287:1835-1837.

Gay SC, Sun L, Maekawa K, Halpert JR, and Stout CD. 2009. Crystal structures of cytochrome P450 2B4 in complex with the inhibitor 1-biphenyl-4-methyl-1H-imidazole: ligand-induced structural response through alphahelical repositioning. Biochemistry 48:4762-4771. 10.1021/bi9003765

Ge R, Pejo E, Cotten JF, and Raines DE. 2013. Adrenocortical suppression and recovery after continuous hypnotic infusion: etomidate versus its soft analogue cyclopropyl-methoxycarbonyl metomidate. Crit Care 17:R20. $10.1186 /$ cc12494

Ge R, Pejo E, Husain SS, Cotten JF, and Raines DE. 2012. Electroencephalographic and hypnotic recoveries after brief and prolonged infusions of etomidate and optimized soft etomidate analogs. Anesthesiology 117:10371043. 10.1097/ALN.0b013e31826d3de2

Hildreth AN, Mejia VA, Maxwell RA, Smith PW, Dart BW, and Barker DE. 2008. Adrenal suppression following a single dose of etomidate for rapid sequence induction: a prospective randomized study. J Trauma 65:573-579. 10.1097/TA.0b013e31818255e8

Ledingham IM, and Watt I. 1983. Influence of sedation on mortality in critically ill multiple trauma patients. Lancet 1:1270.

Li R, Zhang WS, Liu J, Tang M, Yang YY, and Luo NF. 2012. Minimum infusion rates and recovery times from different durations of continuous infusion of fospropofol, a prodrug of propofol, in rabbits: a comparison with propofol emulsion. Vet Anaesth Analg 39:373-384. 10.1111/j.1467-2995.2012.00733.x

Molenaar N, Bijkerk RM, Beishuizen A, Hempen CM, de Jong MF, Vermes I, van der Sluijs Veer G, Girbes AR, and Groeneveld AB. 2012. Steroidogenesis in the adrenal dysfunction of critical illness: impact of etomidate. Crit Care 16:R121. 10.1186/cc11415

Morris C, and McAllister C. 2005. Etomidate for emergency anaesthesia; mad, bad and dangerous to know? Anaesthesia 60:737-740. 10.1111/j.1365-2044.2005.04325.x

Ouellet H, Podust LM, and de Montellano PR. 2008. Mycobacterium tuberculosis CYP130: crystal structure, biophysical characterization, and interactions with antifungal azole drugs. J Biol Chem 283:5069-5080. 10.1074/jbc.M708734200 
282

283

284

285

286

287

288

289

290

291

292

293

294

295

296

297

298

299

300

301
Pejo E, Feng Y, Chao W, Cotten JF, Le Ge R, and Raines DE. 2012. Differential effects of etomidate and its pyrrole analogue carboetomidate on the adrenocortical and cytokine responses to endotoxemia. Crit Care Med 40:187-192. 10.1097/CCM.0b013e31822d7924

Roumen L, Sanders MP, Pieterse K, Hilbers PA, Plate R, Custers E, de Gooyer M, Smits JF, Beugels I, Emmen J, Ottenheijm HC, Leysen D, and Hermans JJ. 2007. Construction of 3D models of the CYP11B family as a tool to predict ligand binding characteristics. J Comput Aided Mol Des 21:455-471. 10.1007/s10822-007-9128-9

Santer P, Pejo E, Feng Y, Chao W, and Raines DE. 2015. Cyclopropyl-methoxycarbonyl Metomidate: Studies in a Lipopolysaccharide Inflammatory Model of Sepsis. Anesthesiology 123:368-376. 10.1097/aln.0000000000000721

Shanmugasundararaj S, Zhou X, Neunzig J, Bernhardt R, Cotten JF, Ge R, Miller KW, and Raines DE. 2013. Carboetomidate: an analog of etomidate that interacts weakly with 11beta-hydroxylase. Anesth Analg 116:1249-1256. 10.1213/ANE.0b013e31828b3637

Tekwani KL, Watts HF, Chan CW, Nanini S, Rzechula KH, and Kulstad EB. 2008. The effect of single-bolus etomidate on septic patient mortality: a retrospective review. West J Emerg Med 9:195-200.

Watt I, and Ledingham IM. 1984. Mortality amongst multiple trauma patients admitted to an intensive therapy unit. Anaesthesia 39:973-981.

Yang J, Kang Y, Wang B, Yang L, Liu J, and Zhang W. 2017. Metabolite-inactive etomidate analogues alleviating suppression on adrenal function in Beagle dogs. Eur J Pharm Sci 99:343-349. 10.1016/j.ejps.2016.12.041 
303

\begin{tabular}{|c|c|c|c|c|c|c|c|c|}
\hline \multicolumn{3}{|c|}{ etomidate } & \multicolumn{3}{|c|}{ ET-26-HCl } & \multicolumn{3}{|c|}{ CPMM } \\
\hline IR & Result & MI & IR & Result & MI & IR & Result & MI \\
\hline 0.33 & + & & 0.65 & + & & 1 & + & \\
\hline 0.30 & + & & 0.59 & - & 0.62 & 0.9 & - & 0.95 \\
\hline 0.27 & - & 0.285 & 0.65 & + & & 1 & + & \\
\hline 0.30 & + & & 0.59 & - & 0.62 & 0.9 & - & 0.95 \\
\hline 0.27 & - & 0.285 & 0.65 & - & & 1 & + & \\
\hline 0.30 & + & & 0.72 & + & & 0.9 & - & 0.95 \\
\hline 0.27 & - & 0.285 & 0.65 & + & & 1 & + & \\
\hline 0.30 & + & & 0.59 & - & 0.62 & 0.9 & - & 0.95 \\
\hline 0.27 & - & 0.285 & 0.65 & + & & 1 & + & \\
\hline 0.30 & + & & 0.59 & - & 0.62 & 0.9 & - & 0.95 \\
\hline \multirow[t]{2}{*}{0.27} & - & 0.285 & 0.65 & + & & & & \\
\hline & & & 0.59 & - & 0.62 & & & \\
\hline \multicolumn{2}{|c|}{$\mathrm{MIR}=0.285$} & & \multicolumn{2}{|c|}{$M I R=0.62$} & & \multicolumn{2}{|c|}{$\mathrm{MIR}=0.95$} & \\
\hline
\end{tabular}

304

305 Notes: A change in the response from negative to positive or positive to negative was defined as a 306 pair, and the stimulation was repeated at different infusion rates until five pairs of responses were 307 recorded. The minimum infusion rate was determined as the average of these five mean values.IR: 308 the infusion rate of each rat $(\mathrm{mg} / \mathrm{kg} \times \mathrm{min})$. MI: mean infusion rate for a pair of responses $(\mathrm{mg} / \mathrm{kg}$ $309 \times \mathrm{min})$. MIR: minimum infusion rate $(\mathrm{mg} / \mathrm{kg} \times \mathrm{min})$. 
A

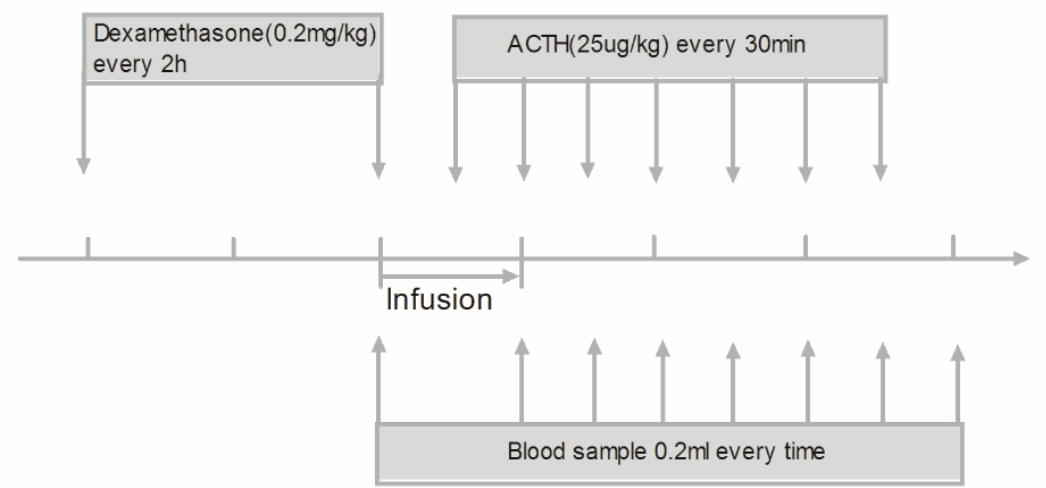

B

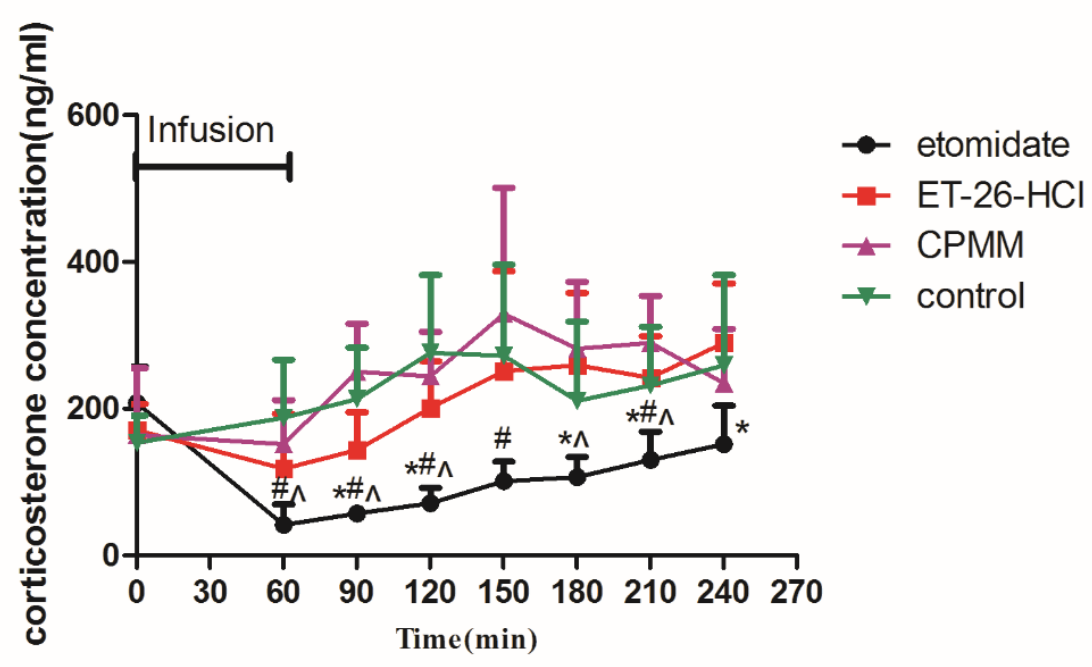

Fig. 1 Determination of serum corticosterone concentrations. (A) Schematic depicting the experimental protocol. Before the hypnotic drug infusion, the first blood sample was drawn as the baseline. Adrenocorticotropic hormone (ACTH) was injected intravenously after 30 min of drug infusion and then once every $30 \mathrm{~min}$ for the duration of the experiment. The second blood sample was collected at the end of the drug infusion, and then blood samples were drawn every 30 $\min$ for 3.5

h. (B) Adrenocortical function as determined by serum corticosterone concentrations after hypnotic drug infusion. ${ }^{*} P<0.05$, for etomidate versus ET-26-HCl; ${ }^{*} P<$ 0.05 for etomidate versus control; $\wedge P<0.05$ for etomidate versus CPMM. Eight rats were used in 
321 each group.

322 